\title{
BLOOD VESSELS EXTRACTION USING MATHEMATICAL MORPHOLOGY
}

\author{
${ }^{1}$ Nidhal Khdhair El Abbadi and ${ }^{2}$ Enas Hamood Al Saadi \\ ${ }^{1}$ Department of Computer Science, University of Kufa, Najaf, Iraq \\ ${ }^{2}$ Department of Computer Science, University of Babylon, Babylon, Iraq
}

Received 2013-07-10, Revised 2013-09-11; Accepted 2013-09-12

\begin{abstract}
The retinal vasculature is composed of the arteries and veins with their tributaries which are visible within the retinal image. The segmentation and measurement of the retinal vasculature is of primary interest in the diagnosis and treatment of a number of systemic and ophthalmologic conditions. The accurate segmentation of the retinal blood vessels is often an essential prerequisite step in the identification of retinal anatomy and pathology. In this study, we present an automated approach for blood vessels extraction using mathematical morphology. Two main steps are involved: enhancement operation is applied to the original retinal image in order to remove the noise and increase contrast of retinal blood vessels and morphology operations are employed to extract retinal blood vessels. This operation of segmentation is applied to binary image of tophat transformation. The result was compared with other algorithms and give better results.
\end{abstract}

Keywords: Blood Vessels, Diabetic, Retina, Segmentation, Image Enhancement

\section{NTRODUCTION}

Diabetes is the commonest cause of blindness in the working age group in the developed world. Patient's sight can be affected by diabetes which causes cataracts, glaucoma and most importantly damage to blood vessels inside the eye, a condition known as "diabetic retinopathy". Diabetic retinopathy is a critical eye disease which can be regarded as manifestation of diabetes on the retina. The screening of diabetic patients for the development of diabetic retinopathy can potentially reduce the risk of blindness in these patients by $50 \%$.

The leading cause of blindness in working age people is due to diabetic omplications. Diabetes affects the vascular system and the potential consequence of this for the eye is progressive death of the retina due to the loss of the blood supply. Diabetic Retinopathy (DR) is one of the leading causes of blindness among people suffering from diabetes. It is observed that about $2 \%$ of the patients affected by this disorder are blind and $10 \%$ undergo vision degradation after 15 years of diabetes. DR patients perceive no symptoms until visual loss develops which happens usually in the later disease stages, when the treatment is less effective (Honale and Kapse, 2012).

The detection of blood vessels is a major problem in the automatic processing of retinal images. On the one hand, the vessels have certain properties such as diameter which may be the key indicators in the evolution of certain retinopathies. On the other hand, the vascular tree is a principal element in the eye, its detection is crucial for automated analysis of retinal images, because it allows locating the papilla which can be defined as its origin, the macula by its absence. The appearance of the retinal blood vessels is an important diagnostic indicator of various clinical disorders of the eye and the body. Retinal blood vessels have been shown to provide evidence in terms of change in diameter, branching angles, or tortuosity, as a result of ophthalmic disease (Fraz et al., 2013).

Retinal vessels are affected by many diseases. In conditions such as diabetic retinopathy, the blood vessels Corresponding Author: Nidhal Khdhair El Abbadi, Department of Computer Science, University of Kufa, Najaf, Iraq 
often show abnormalities at early stages. Changes in retinal blood vessels are also associated with hypertension and other cardio-vascular conditions. Common structural changes associated with vessels include changes in width, disappearance of vessels due to occlusion and growth of new vessels i.e., neovascularization. For instance, it is shown that the retinal arteries dilate by about $35 \%$ in cases with hypertension. Age and hypertension can also cause changes in the bifurcation geometry of retinal vessels.

Current methods of detection and assessment of diabetic retinopathy are manual, expensive and require trained ophthalmologists. Retinal blood vessel morphology can be an important indicator for many diseases such as diabetes, hypertension and arteriosclerosis. The measurement of geometrical changes in veins and arteries can be applied to a variety of clinical studies. Two major problems in the segmentation of retinal blood vessels are the presence of a wide variety of vessel widths and the heterogeneous background of the retina (Dey et al., 2012).

\subsection{Related Works}

Kumari and Suriyanarayanan (2010) extracted the blood vessels using Wiener filter and morphological operation opening and closing. The peak signal to noise ratio is calculated for both the methods and are compared. The edge of the blood vessels are detected by applying Laplacian and Gaussian operators and the thinning of blood vessel is done using morphological operator and smoothened for better clarity in the extracted blood vessel.

Segment retinal blood vessels method presented by (Xu and Luo, 2010) to overcome the variations in contrast of large and thin vessels. This method uses adaptive local thresholding to produce a binary image, then extract large connected components as large vessels. The residual fragments in the binary image including some thin vessel segments (or pixels), are classified by Support Vector Machine (SVM). The tracking growth is applied to the thin vessel segments to form the whole vascular network.

Badsha et al. (2013) present automatic method to extract the retinal blood vessel. The proposed method comprises several basic image processing techniques, namely edge enhancement by standard template, noise removal, thresholding, morphological operation and object classification. The proposed method has been tested on a set of retinal images.

Kaba et al. (2013) introduced an automated method to segment blood vessels in fundus retinal images. The method could be used to support a non-intrusive diagnosis in modern ophthalmology for early detection of retinal diseases, treatment evaluation or clinical study. This method combines the bias correction to correct the intensity in homogeneity of the retinal image and a matched filter to enhance the appearance of the blood vessels. The blood vessels are then extracted from the matched filter response image using the Expectation Maximization algorithm.

\subsection{Segmentation of Retinal Vasculature Issues}

The retinal vasculature is composed of the arteries and veins with their tributaries which are visible within the retinal image.

The segmentation and measurement of the retinal vasculature is of primary interest in the diagnosis and treatment of a number of systemic and ophthalmologic conditions. The accurate segmentation of the retinal blood vessels is often an essential prerequisite step in the identification of retinal anatomy and pathology.

There are some factors that hinder vascular segmentation such as:

- Vessels are obviously not all with the same size, shape, or color

- The contrast can sometimes be quite low; and the vessel color can be close to that of the background

- Some background features (e.g., underlying choroidal structures, or the nerve fiber layer) have similar attributes to vessels

- Vessel crossings and bifurcations may confuse some techniques

- The edge of the optic disk can be wrongly egmented as a vessel

\section{MATERIALS AND METHODS}

The detection of blood vessels is a major problem in the automatic processing of retinal images. On the other hand, the vessels have certain properties such as diameter which may be the key indicators in the evolution of certain retinopathy.

The segmentation of blood vessels is the best technique to find the ridge lines in an image. In this case, ridge lines correspond to skeleton of blood vessels, where it is not necessary to extract the whole network but only to find the skeleton.

A proposed algorithm presented in this study for automatically detecting of blood vessels in the retinal images. The algorithm was developed based on fundus 
images. The main goal of the study is detection of the blood vessels. The representation of an image in RGB color space allows to studying separately the different channels of the spectral response. The three channels are (red, green and blue).

\subsection{Preprocessing}

The main step in this study is to extract the blood vessels by using color fundus image. Fundus image is an RGB color image, in general RGB images consist of three channels (red, green and blue) and this feature will investigated in this study for localization of the blood vessels. This can be accomplished by separation the retina image to three channels and using only one of them (Green channel), the blue channel is characterized by low contrast and does not contain much information. The vessels are visible in the red channel but this channel usually contains too much noise or it is simply saturated, since most of the features emit a signal in the red channel. While the green component of the color retina image gives the best result in the contrast of blood vessels (darker blood vessels on a bright background). Therefore, the green channel of the image is used in the automated analysis of fundus images as shown in Fig. 1.

\subsection{Enhencement}

Retinal images after acquisition are generally noisy, low contrast and non-uniform illumination and therefore we apply some algorithms to enhance image and noise reduction by increase contrast and filtering. All the components in the green channel will be labeled by using image label algorithm, this achieved by scan all the image pixels, assigning preliminary labels to nonzero pixels and recording label equivalences in a union-find table. Then resolve the equivalence classes using the union-find algorithm (The Union-Find algorithm is used for maintaining a number of nonoverlapping sets from a finite universe of elements). Finally relabeled the pixels based on the resolved equivalence classes.

For all labeled regions in the green channel of retinal image we compute area and then find the max area among all labeled regions, boarder the max area with white board as shown in Fig. 2.

To solve the problems of the false detection of the contour in the retinal as blood vessels due to the presence of atrophy in the border of the retinal (There is a similarity between border of the retinal and blood vessels). This resolved by removing the background for the resulted image (Fig. 2) by convert the background to white color (every pixel outside the white board changed to white color) as shown in Fig. 3.

A contrast-limited adaptive histogram equalization "CLAHE" was applied for contrast enhancement. "CLAHE" operates on small regions in the image. The contrast of each small region is enhanced with histogram equalization.

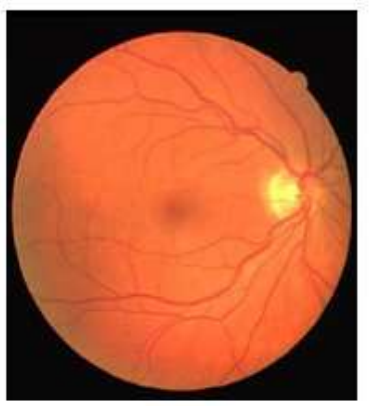

(A)

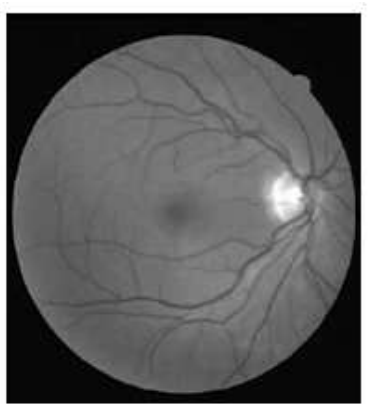

(B)
Fig. 1. (A) A typical color retinal image, (B) Green channel of image in A

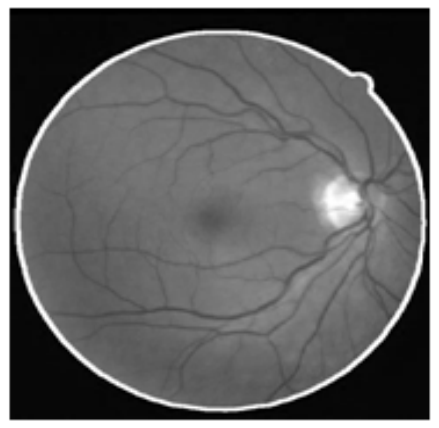

Fig. 2. Boarding the retinal with white board

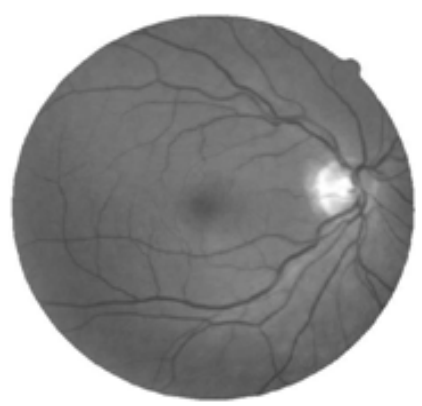

Fig. 3. Retinal image after removing the background 
The techniques of "CLAHE" are well-known for their local contrast enhancement of the images and facilitate the task of many physicians before image analysis. Enhancement constitutes the first step towards automatic analysis of retinal images. "CLAHE" technique applied to the image after removing background, this make the image well contrasted as shown in Fig. 4A.

After performing the equalizations, a Gaussian filtering operation was then applied to reduce noise, this step accomplished by two steps, the first one is the filtering operation carried out by removing bright details smaller than a threshold by applying area opening (i.e., small bright details) and the second step by applying a Gaussian filter for noise reduction:

$$
\mathrm{G}(\mathrm{x}, \mathrm{y})=\frac{1}{2 \pi \sigma^{2}} \exp \left(\frac{\mathrm{x}^{2}+\mathrm{y}^{\mathrm{z}}}{\sigma^{2}}\right)
$$

Application of combined the opening operation and Gaussian filter try to reduce all details that are not belonging to vessels but to some noise in the image. Filtering result is shown in Fig. $\mathbf{4 B}$.

\subsection{Highlight the Vascular Network}

In order to highlight the vascular network we perform two steps.

\subsection{Top-Hat Transforms}

In mathematical morphology and digital image processing, top-hat transform is an operation that extracts small elements and details from given images. There exist two types of top-hat transform: The white top-hat transform which defined as the difference between the input image and its opening by some structuring element; The black top-hat transform which defined dually as the difference between the closing and the input image. Tophat transforms are used for various image processing tasks, such as feature extraction, background equalization, image enhancement and others.

Once the image has been filtered, a preliminary detection of blood vessels is carried out. The top-hat transformation applied on the filtered image with a disk structure element with a size large enough to fill all the holes in blood vessels. The top hat transformation is then performed by a closing operation, which is defined by the following equation:

$$
T_{b}(f)=f \bullet b-f
$$

where, $T_{b}(f)$ is the top-hat transformation, $(\bullet)$ is the closing operation, $b$ is the structuring element and $f$ is the filtered image.

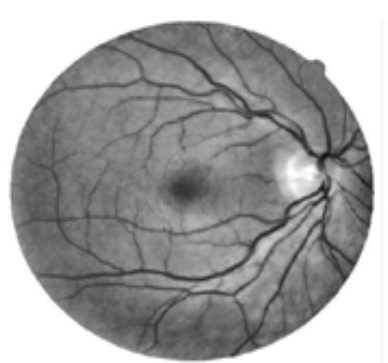

(A)

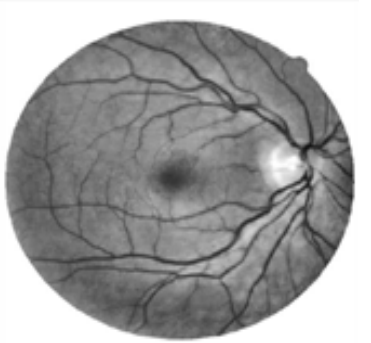

(B)
Fig. 4. Enhance and filtered images, (A) Adaptive Histogram Equalization (AHE), (B) Filtered image

The top-hat returns an image, containing the "objects" or "elements" that are "smaller" than the structuring element and are darker than their surroundings.

The size, or width, of the elements that are extracted by the top-hat transforms can be controlled by the choice of the structuring element b. The bigger structuring element, the larger the elements extracted.

The top-hat transforms is shown in Fig. $\mathbf{5 A}$, the blood vessels appear as clear elongated objects while the background spreads to be black.

\subsection{Adjust Image Intensity}

To increase the contrast of the output image result from top-hat transformation we perform the adjustment on the resulted image from top-hat transformation (i.e., adjust image in intensity value or color map) as shown in Fig. 5B.

Now the resulted image will be thresholding to remove all unnecessary details, the result image is binary image shown in Fig. 5C.

Details extracted by this technique contain not only blood vessels but also other details from the background. In this image, we see a large difference in contrast between thick and small blood vessels. It is clear that thresholding methods are not suitable enough to extract the vascular network. To solve the problem of segmentation, we use the morphology filtering.

\subsection{Morphological Image Processing}

Binary images may contain numerous imperfections. In particular, the binary regions produced by simple thresholding are distorted by noise and texture. Morphological image processing pursues the goals of removing these imperfections by accounting for the form and structure of the image. 


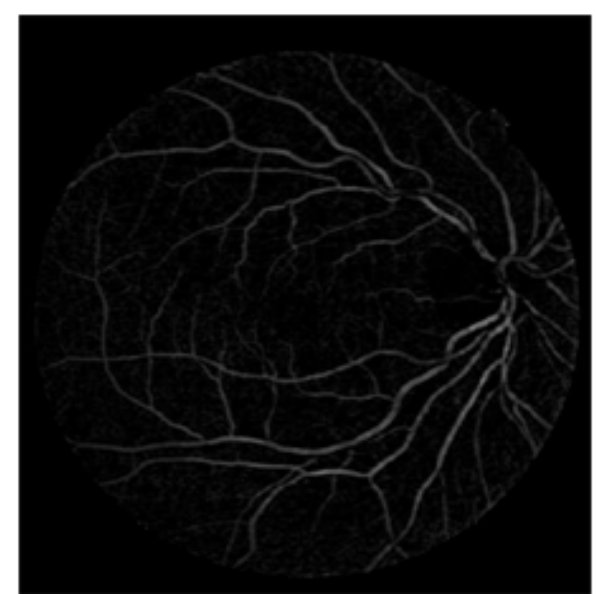

(A)

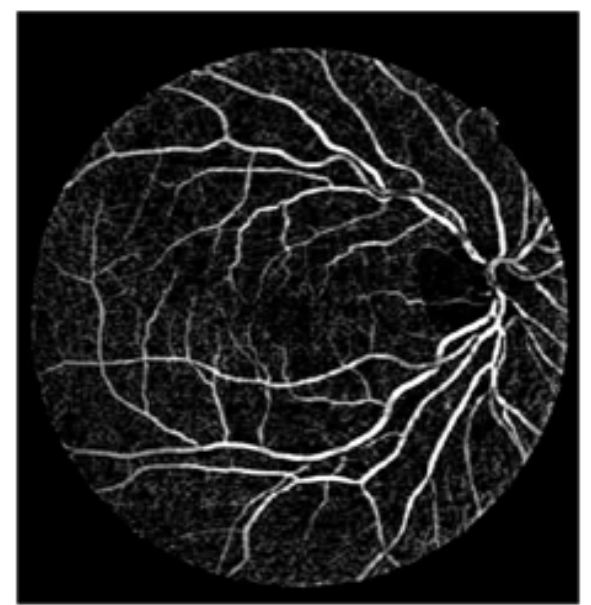

(B)

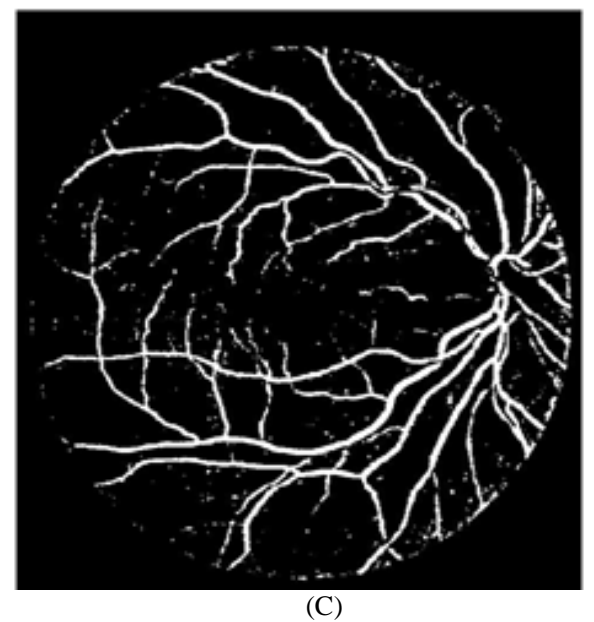

Fig. 5. (A) Top-hat transformation, (B) Adjust image intensity, (C) image after threshold

\subsection{Morphological Filtering}

Morphological filtering is conducted by considering compound operations like opening and closing as filters. They may act as filters of shape. These operations can filter out any details that are smaller in size than the structuring element from an image, e.g., opening is filtering the binary image at a scale defined by the size of the structuring element. Only those portions of the image that fit the structuring element are passed by the filter; smaller structures are blocked and excluded from the output image. The size of the structuring element is most important to eliminate noisy details but not to damage objects of interest. We'll use morphological techniques called "opening by reconstruction" and "openingclosing by reconstruction" to "clean" up the image.

\subsection{Opening by Reconstruction}

The normal morphological opening is erosion followed by dilation. The erosion "shrinks" an image according to the shape of the structuring element, removing objects that are smaller than the shape. Then the dilation step "re-grows" the remaining objects by the same shape, while opening-by-reconstruction is (1) erosion followed by (2) a morphological reconstruction as shown in Fig. 6A.

\subsection{Opening-Closing by Reconstruction}

Now use dilatation to the opening by reconstruction followed by morphological reconstruction to the complement of the output of dilation and complement of the output result from opening by reconstruction, finally, complement the output of morphological reconstruction as shown in Fig. 6B.

Reconstruction based opening and closing are more effective than standard opening and closing to removing small blemishes without affecting the overall shapes of the objects. Following the opening with a closing can remove the dark spots and stem marks.

\subsection{Labeling Regions}

By this step all the components (regions) in the binary image resulted from opening-closing by reconstruction will be labeled by using image label algorithm as describe previously. This will help to extract blood vessels network only by recognize the blood vessels from the other similar objects. 


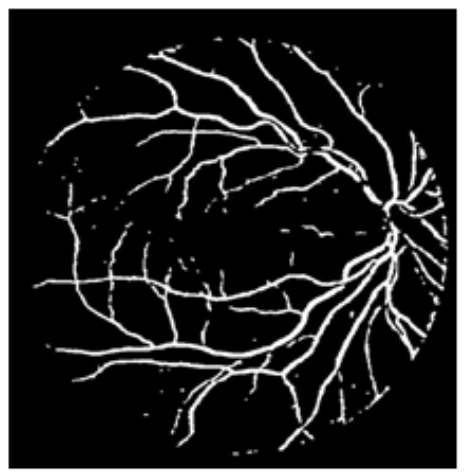

(A)

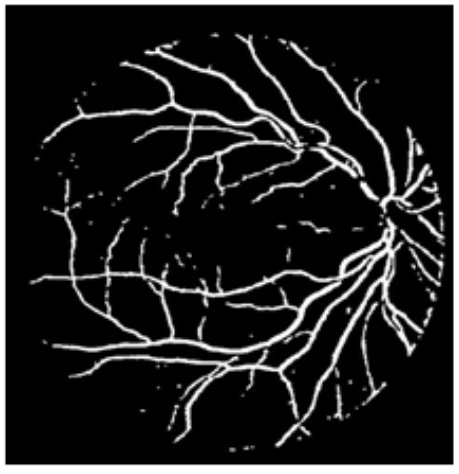

(B)

Fig. 6. (A) Opening by reconstruction, (B) Opening-closing by reconstruction

The process of recognizing the blood vessels depending on the shape of these components, the blood vessels not have circular shape. Then any circles shapes will be removed. This is achieved by determine the circularity of image regions from the relation:

$$
\text { Circularity }=4 \times \mathrm{pi} \times\left(\text { area } / \text { perimeter }^{2}\right)
$$

Area and perimeter is the area and perimeter of the interested labeled region which counting the circularity for it.

From the experiment, we found that if the circularity $>=0.2$, then the region is not blood vessels, therefore should be removed it. The result showed in Fig. 7.

\subsection{Watershed Transform}

The watershed constitutes one of the main concepts of Mathematical Morphology. The watershed transform can be classified as a region-based segmentation approach, which can be applied to gray scale image to solve a variety of image segmentation problems. In our case, we apply this technique in order to extract blood vessels from image background.

In the following, we present the result of algorithm that we have developed to compute segmentation of retinal image. We calculate the distance transform of binary image obtained from previous sections. The distance transform of a binary image is the distance of every pixel to the nearest nonzero-value pixel. Figure 8A shows the distance transform applied on binary image showed in Fig. 7.

Finally, we applied a watershed transformation to the distance function to obtain the watershed ridge line Fig. 8B. The centerline of blood vessels is obtained by calculate skeleton of the binary image result from watershed ridge line to obtain skeleton of retinal network. This operation completes the segmentation as shown in Fig. 9.

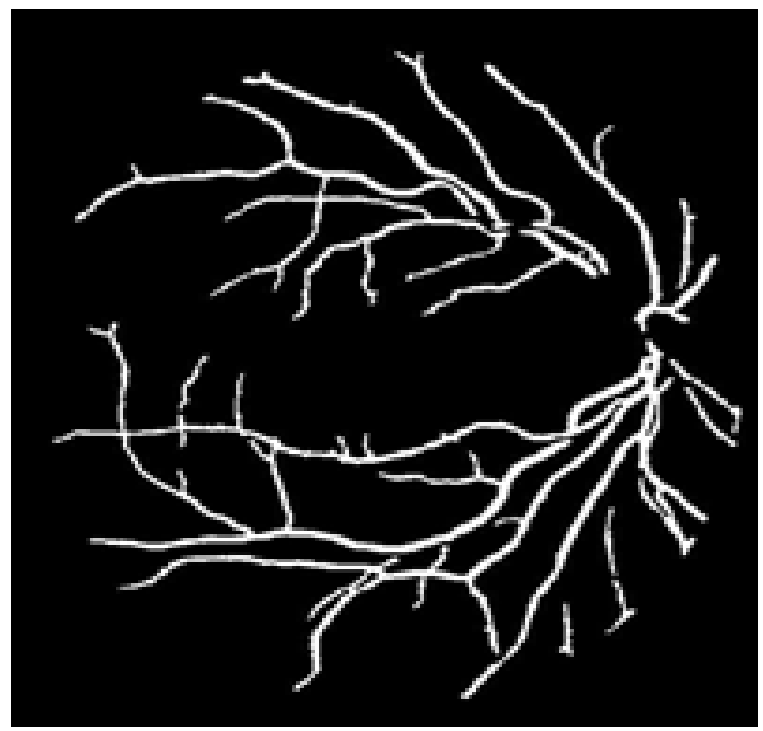

Fig. 7. Blood vessel network

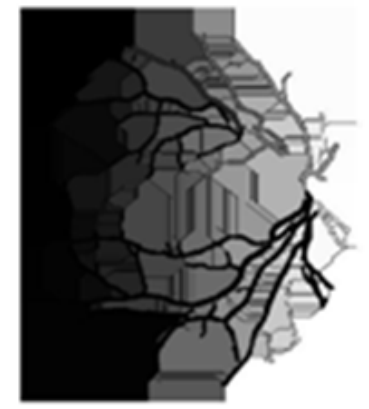

(A)

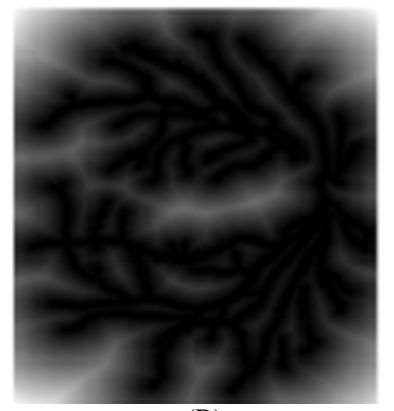

(B)
Fig. 8. (A) Distance function, (B) Watershed ridge lines 


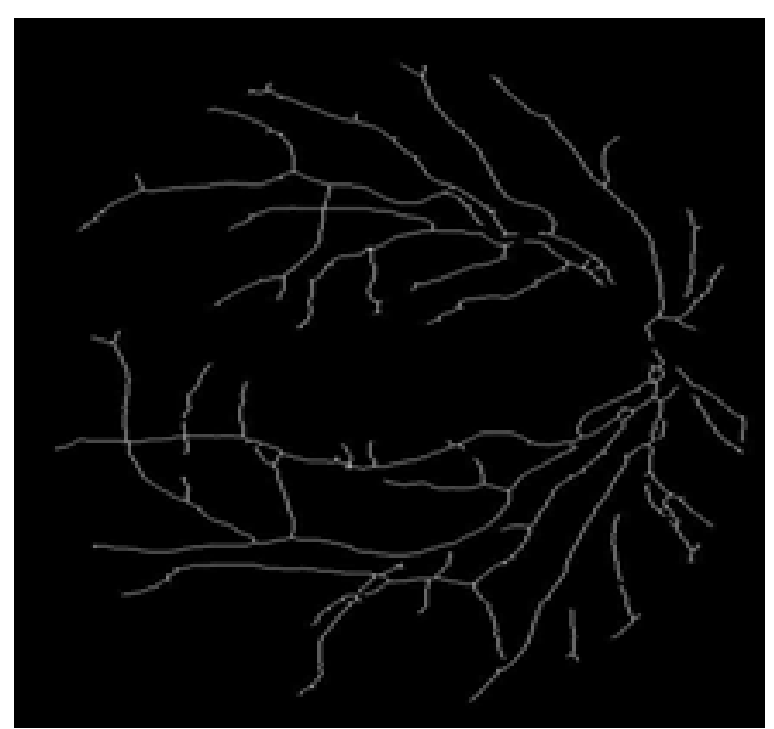

Fig. 9. Skeleton of retinal network

Table 1. Comparing PSNR for different methods

\begin{tabular}{llll}
\hline Methods & $\begin{array}{l}\text { Wiener } \\
\text { filter method }\end{array}$ & $\begin{array}{l}\text { Morphological } \\
\text { method }\end{array}$ & $\begin{array}{l}\text { Proposed } \\
\text { method }\end{array}$ \\
\hline PSNR & 5.6861 & 5.8025 & 8.1501 \\
\hline
\end{tabular}

\section{RESULTS AND DISCUSSION}

The PSNR value for the current method is calculated and compared with the PSNR resulted from other methods (Kumari and Suriyanarayanan, 2010) as shown in the Table 1.

It is clear this method more depandable and gives promised results. This is due to the suggested methods of removing the noise and determined the cicularity.

\section{CONCLUSION}

This study introduced new method to extract the blood vessels, in this study many algorithms used to achieve the best result, like the top-hat transformation, opening by reconstruction, opening-closing by reconstruction, erosion, dilation, watershed.

Some properties recognize this study:

- It resolved the problem of false detection of the contour for the retinal which detects as blood vessels, this is due to the presence of atrophy in the border of the retinal
- In the previous works they used the top-hat transformation to highlight the vascular network, while in this algorithm we suggested to use the adjustment on the resulted image from top-hat transformation to highlight vascular network and increase the image contrast

- In this study we use new way to remove all the non blood vessels by determine the circularity of shapes or regions

- In general the study recognizes most of blood vascular and gives result better than the result in other papers

\section{REFRENCES}

Badsha, S., A.W. Reza, K.G. Tan and K. Dimyati, 2013. A new blood vessel extraction technique using edge enhancement and object classification. J. Digital Imag. DOI: 10.1007/s10278-013-9585-8

Dey, N., A.B. Roy, M. Pal and A. Das, 2012. FCM based blood vessel segmentation method for retinal images. Int. J. Comput. Sci. Netw.

Fraz, M.M., A. Basit and S.A. Barman, 2013. Application of morphological bit planes in retinal blood vessel extraction. J. Digit. Imag., 26: 274-286. DOI: $\quad 10.1007 / \mathrm{s} 10278-012-9513-3, \quad$ PMID: 22832895

Honale, S.S. and V.S. Kapse, 2012. A review of methods for blood vessel segmentation in retinal images. Int. J. Eng. Res. Technol., 1: 1-6.

Kaba, D., A.G. Salazar-Gonzalez, Y. Li, X. Liu and A. Serag, 2013. Segmentation of retinal blood vessels using gaussian mixture models and expectation maximization. Proceedings of the 2nd International Conference on Health Information Science, Mar. 25-27, Springer Berlin Heidelberg, London, UK., pp: 105-112. DOI: 10.1007/978-3642-37899-7_9

Kumari, V.V. and N. Suriyanarayanan, 2010. Blood vessel extraction using wiener filter and morphological operation. Int. J. Comput. Sci. Emerg. Technol., 1: 7-11.

$\mathrm{Xu}, \mathrm{L}$. and S. Luo, 2010. A novel method for blood vessel detection from retinal images. Biomed. Eng., 9: 14-14. DOI: $10.1186 / 1475-925 X-9-14$ 